\title{
Estresse em cuidadores de crianças com transtorno do déficit de atenção e hiperatividade
}

\author{
Ana Maria Del Bianco Faria \\ Centro Universitário UNIFAFIBE, SP, Brasil \\ Carmen Lúcia Cardoso \\ Universidade de São Paulo, SP, Brasil
}

\begin{abstract}
Resumo
Objetivou-se avaliar o estresse, auto-percepção de estresse e de fatores estressantes em cuidadores de crianças com Transtorno do Déficit de Atenção e Hiperatividade (TDAH). Participaram 40 cuidadores, divididos em: Grupo clínico (G1) - 20 cuidadores de crianças diagnosticadas com TDAH; Grupo de comparação (G2) - 20 cuidadores de crianças sem TDAH. Utilizaram-se os instrumentos: Questionário de Capacidades e Dificuldades, Inventário de Sintomas de Stress para Adultos de Lipp, Questionário de Auto-Percepção de estresse. Os resultados apontaram percentual de cuidadores com indicadores de estresse significativamente maior no G1 do que encontrado no G2 ( $p=0,001)$. No G1 houve maior percepção de estresse no convívio com a criança $(p<0,001)$, no cuidado com a criança $(p<0,001)$ e maior percepção de estresse $(p=0,003)$ quando comparados ao $\mathrm{G} 2$. Destaca-se a necessidade de cuidado ao cuidador, pois a vivência de estresse afeta a qualidade de vida, bem como a relação e o vínculo entre este e a criança.

Palavras-chave: Transtorno do déficit de atenção e hiperatividade; Cuidadores; Estresse.
\end{abstract}

\section{Stress in caregivers of children with attention-deficit hyperactivity disorder}

\begin{abstract}
This study aimed to assess stress and self-perception of stress and stressful factors in caregivers of children with attention deficit hyperactivity disorder (ADHD). Participants were 40 caregivers, divided into: clinical group (G1) - 20 caregivers of children diagnosed with ADHD; comparison group (G2) - 20 caregivers of children without ADHD. The instruments used included the Strengths and Difficulties Questionnaire, Lipp's Inventory of Stress Symptoms for Adults and the self-perceived Stress Questionnaire. Results point to a percentage of caregivers with stress indicators significantly higher in G1 than that found in G2 $(p=0.001)$. In $\mathrm{G} 1$, there was a greater perception of stress in the life with the child $(p<0.001)$, in the care of the child $(p<0.001)$, and an even greater perception of stress $(p=0.003)$ when compared to $\mathrm{G} 2$. The need to care for caregiver is noteworthy, since experiencing stress affects quality of life and the relationship and bonding between caregiver and child.
\end{abstract}

Keywords: Attention-deficit hyperactivity disorder; Caregivers; Stress.

\section{Estrés en cuidadores de niños con trastorno del déficit de atención e hiperactividad}

\section{Resumen}

El objetivo fue evaluar el estrés, la autopercepción del estrés y factores estresantes en cuidadores de niños con Trastorno del Déficit de Atención e Hiperactividad (TDAH). Participaron 40 cuidadores divididos en: Grupo clínico (G1) - 20 cuidadores de niños diagnosticados con TDAH; Grupo de comparación (G2) - 20 cuidadores de niños sin TDAH. Se utilizaron los instrumentos: Cuestionario de Capacidades y Dificultades, Inventario de Síntomas de Stress para Adultos de Lipp y Cuestionario de Autopercepción de Estrés. Los resultados señalaron el porcentual de cuidadores con indicadores de estrés significativamente mayor en el G1 que el encontrado en el G2 ( $p=0,001)$. En el G1 hubo mayor percepción de estrés en la convivencia con el niño $(p<0,001)$, en el cuidado del niño $(p<0,001)$ y, aun, mayor percepción de estrés $(p=0,003)$ cuando comparado al G2. Se hace necesario cuidar del cuidador, pues la experiencia de estrés afecta la calidad de vida, la relación y el vínculo entre cuidador y niño.

Palabras clave: Trastorno del déficit de atención e hiperactividad; Cuidadores; Estrés. 


\section{Introdução}

OTranstorno do Déficit de Atenção e Hiperatividade (TDAH) é uma desordem neuropsicobiológica que se manifesta, essencialmente, por níveis clinicamente significativos de desatenção, hiperatividade e impulsividade, que devem estar presentes em mais de um contexto de vida da criança, afetando áreas do funcionamento adaptativo (American Psychiatric Association, 2002). Caracteriza-se como distúrbio heterogêneo, com etiologia multifatorial, dependendo de fatores genéticos, adversidades biológicas e psicossociais (Rodhe et al., 1998). Estima-se que atualmente o TDAH seja um dos transtornos mentais mais frequentemente diagnosticados entre as crianças, inclusive entre as brasileiras (Petresco et al, 2014).

A prática diagnóstica e terapêutica do TDAH é complexa e impõe um desafio aos profissionais de saúde e educação. Atualmente, no Brasil, estudo aponta a prevalência de $6,01 \%$ do transtorno, com predomínio do gênero masculino (75\%) (Biscegli et al., 2013). No entanto, uma revisão de literatura conduzida por Hora, Silva, Ramos, Pontes e Nobre (2015) mostra uma ampla variação nas estimativas de prevalência em diferentes países, indicando desde taxas baixas $(2,7 \%)$ até muito elevadas $(31,1 \%)$ tendo em vista os critérios metodológicos e diagnósticos adotados. De acordo com Santos e Vasconcelos (2010), o diagnóstico deve ser refeito a cada semestre, sugerindo aspecto dinâmico e transitório do transtorno. Além disso, durante o diagnóstico, é importante se recorrer a várias fontes de informação (pais, demais familiares e escola), pois somente assim será possível observar o real impacto do comportamento apresentado pelo indivíduo em diferentes contextos e controlar o risco de uma avaliação incorreta (Oliveira \& Albuquerque, 2009).

Em relação ao tratamento, a literatura aponta que atualmente houve um aumento no uso da prática medicamentosa, parecendo haver privilégio desta terapêutica no cuidado ao transtorno (Caliman, 2008). No entanto, o TDAH é um fenômeno complexo e multideterminado, que influencia e é influenciado pelo contexto familiar, portanto exige um cuidado global e não apenas uma atenção voltada à seus aspectos biológicos (Pereira \& Silva, 2011).

Alguns trabalhos indicam associação entre o ambiente familiar e o TDAH. Crianças com o transtorno apresentaram famílias com o ambiente menos organizado e com maiores conflitos (Schroeder \& Kelley, 2009) além de um pior funcionamento familiar geral, como no planejamento de atividades, desentendimentos, relacionamento afetivo, entre outros (Pires, Silva, \& Assis, 2012). No entanto, cabe ressaltar a dificuldade de afirmar se a disfunção familiar é a causa ou o efeito do TDAH, pois existem dificuldades nos vínculos entre as crianças e os cuidadores primários, mantidas através de processos de retroalimentação (D'avila-Bacarji et al., 2005). Estudo realizado por Bargas e Lipp (2013), que avaliou a influência do estresse e do estilo parental sobre o estresse de crianças portadores de TDAH, apontou alto nível de estresse nas crianças e suas mães, além de estilo parental materno classificado como de risco para o desenvolvimento de comportamentos antissociais e que a sintomatologia do estresse materno tem relação com o subtipo de TDAH. Nunes e Werlang (2008) apontam a necessidade de se entender o indivíduo como parte de um sistema, com elementos que interagem entre si, influenciando cada parte e sendo por elas influenciados.

Nesse sentido, atender às necessidades básicas do indivíduo com TDAH passa a fazer parte do cotidiano dos familiares, o que inclui coordenar suas atividades diárias, acompanhá-los ao serviço de saúde, lidar com seus comportamentos problemáticos, fornecer-lhes suporte social, entre outros (Marini, Martins, Vigãno, Filho \& Pontes, 2010). Assim, as dificuldades em lidar com a situação resultam numa sobrecarga para a família, estudos apontam a associação do estresse a diferentes contextos, entre estes o cuidado a um indivíduo em sofrimento (Del Bianco Faria \& Cardoso, 2010; Santos \& Cardoso, 2015).

O termo estresse foi definido por Selye (1956), como uma quebra da homeostase interna, eliciada por um estressor que consiste em qualquer evento ou situação do ambiente que altere as condições internas, exigindo esforço de adaptação. $\mathrm{O}$ autor diferenciou e descreveu três fases das alterações físicas e químicas produzidas pelo estresse: fase de alerta, fase de resistência e fase de exaustão. $\mathrm{O}$ estresse é um mecanismo normal e benéfico ao homem, se compreendido e controlado. No entanto, a perda de controle e cronicidade podem gerar um estado de exaustão, tanto físico quanto psíquico e, neste momento, várias doenças podem se manifestar (Selye, 1983; Tabaquim \& Marquesini, 2013).

A presença de uma doença na infância é considerada um agente estressor capaz de atingir o desenvolvimento normal da criança e as relações sociais de seu sistema familiar (Silva \& Correa, 2006). Estudos mostram que ter uma criança com TDAH na família esteve associado ao estresse familiar (Bellé, Andreazza, Ruschel, \& Bosa, 2009; Yousefia, Far, \& Abdolahian, 2011). No contexto brasileiro, Bellé et al. (2009) apontam a necessidade do desenvolvimento de estudos a respeito de aspectos psicossociais dos cuidadores de crianças com diagnóstico de TDAH, pois a vivência de estresse aliada às exigências relativas à função de 
cuidar impactam o funcionamento familiar e podem afetar a qualidade de vida do cuidador, bem como sua capacidade de acolhimento à criança.

A relevância do contexto social em que a criança vive pode funcionar tanto como desencadeador ou potencializador de diversas perturbações quanto pode conter, ou mesmo minimizar as consequências dos transtornos mentais já desenvolvidos. Assim, conhecer a família que cuida e identificar suas forças/ potencialidades e necessidades/dificuldades permite um olhar ampliado para a questão do transtorno, e possibilita instrumentalizar a equipe de saúde no tratamento à criança e ao familiar.

Nesse contexto, o objetivo deste estudo foi avaliar o nível de estresse, a autopercepção de estresse e de fatores estressantes em cuidadores primários de crianças com diagnóstico de TDAH, comparando-os com grupo de cuidadores de crianças sem diagnóstico de doença e/ ou tratamento psicológico, psiquiátrico e neurológico.

\section{Método}

\section{Participantes}

A pesquisa foi realizada com 40 cuidadores primários de crianças, com idades entre 6 e 12 anos, divididos em: o grupo clínico $(\mathrm{G} 1-\mathrm{n}=20)$ e o grupo de comparação $(\mathrm{G} 2-\mathrm{n}=20)$. Adotou-se como definição de cuidador primário aquele que tinha a principal responsabilidade no fornecimento de ajuda e cuidados, prestados no domicílio, à pessoa acometida (Stone, Cafferata \& Sangl, 1987).

Os critérios de inclusão dos participantes foram: ser o principal provedor de cuidados à criança (com idade entre 6 e 12 anos) e residir no mesmo local que a criança. Especificamente para o Grupo 1: a criança estar atualmente em atendimento na unidade de saúde; ter confirmação de diagnóstico médico (psiquiátrico ou neurológico) de TDAH, sem, no entanto, ter iniciado o tratamento medicamentoso; e ter obtido no Questionário de Capacidades e Dificuldades (SDQ), aplicado ao cuidador, na escala específica para hiperatividade pontuação entre 7-10 e não ter outros diagnósticos médicos. E, especificamente, para o Grupo 2: criança sem o diagnóstico de TDAH; sem histórico de doença crônica, atendimento psicológico, psiquiátrico e/ou neurológico já diagnosticado ou tratado; e obter no $S D Q$ escore, em cada escala específica e no total das dificuldades, dentro da faixa de normalidade.

As crianças dos dois grupos foram pareadas em relação à idade, sexo e renda familiar, sendo que $80 \%$ das crianças eram do sexo masculino. Em relação à idade, $30,0 \%$ das crianças tinham entre 6 e 7 anos, $40,0 \%$ entre 8 e 9 anos e $30,0 \%$ entre 10 e 12 anos.
Todas as crianças do Grupo 1 (grupo com diagnóstico de TDAH), receberam atendimento médico (neurológico ou psiquiátrico), oito delas (40\%) receberam tratamento psicológico e duas (20\%) psicopedagógico, e ainda $70 \%$ delas iriam iniciar o uso de medicamentos, que havia sido prescrito pelos médicos.

O perfil sociodemográfico dos cuidadores participantes encontra-se na Tabela 1.

\section{Instrumentos para coleta de dados}

- Questionário de Capacidades e Dificuldades - versão brasileira (Fleitlich-Bilyk, 2002) validada do Strenghts and Difficulties Questionnaire (SDQ) (Goodman, 1997), é um questionário breve, composto por 25 itens, utilizado para o rastreamento de problemas de saúde mental em crianças e adolescentes, com faixa etária entre 4 e 16 anos. Os itens focalizam o comportamento da criança, suas emoções e relacionamento com outras pessoas, podem ser respondidos pelos pais/cuidadores e/ou professores, e são agrupados em 5 escalas, a saber: hiperatividade/déficit de atenção, sintomas emocionais, problemas de conduta, problemas de relacionamento e comportamento pró-social. Cada escala contém 5 itens e cada item pode ser respondido como "falso", "mais ou menos verdadeiro" ou "verdadeiro". Para cada uma das 5 escalas, a pontuação pode variar de zero a 10 . As crianças podem ser classificadas em uma de três categorias (normal, limítrofe e anormal) para cada uma das escalas e de acordo com a pontuação total. Posteriormente às escalas, interroga-se também sobre o impacto que os sintomas relatados têm sobre a vida da criança e de sua família. A versão original do $S D Q$ possui propriedades psicométricas adequadas, com coeficiente alfa de Cronbach de 0,73.

- Inventário de Sintomas de Stress para Adultos de Lipp (ISSL) - validado por Lipp (2000) para avaliar as manifestações de estresse, os tipos de sintomatologia e a fase do estresse em que o sujeito se encontra. O ISSL apresenta três quadros que contêm sintomas físicos e psicológicos de cada fase do estresse. A primeira parte do inventário contém uma lista de 15 sintomas de estresse na qual o participante assinala os apresentados nas últimas 24 horas, na segunda parte assinala problemas experimentados na última semana em uma lista de 15 sintomas e na terceira parte, por fim, o respondente deve assinalar, dentre 23 sintomas, os experimentados no último mês. Apresentou coeficiente alfa de Cronbach de 0,91 .

- Questionário de Autopercepção em Relação ao Estresse - questionário desenvolvido pelas próprias pesquisadoras buscando abarcar a percepção dos cuidadores acerca de si mesmos e de possíveis fatores estressantes, tanto no convívio com a criança 
TABELA 1

Caracterização dos cuidadores participantes do Grupo 1 e do Grupo 2 em função das variáveis sócio-demográficas

\begin{tabular}{|c|c|c|c|c|c|}
\hline \multirow{2}{*}{ Variável } & \multirow{2}{*}{ Especificação } & \multicolumn{2}{|c|}{ Grupo $1(n=20)$} & \multicolumn{2}{|c|}{ Grupo $2(n=20)$} \\
\hline & & $F$ & $\%$ & $f$ & $\%$ \\
\hline \multirow{2}{*}{ Sexo } & Feminino & 20 & 100,0 & 20 & 100,0 \\
\hline & Masculino & 0 & - & 0 & - \\
\hline \multirow{4}{*}{ Faixa etária em anos } & $21-30$ anos & 7 & 35,0 & 6 & 30,0 \\
\hline & $31-40$ anos & 4 & 20,0 & 8 & 40,0 \\
\hline & $41-50$ anos & 4 & 20,0 & 4 & 20,0 \\
\hline & $51-60$ anos & 5 & 25,0 & 2 & 10,0 \\
\hline \multirow{2}{*}{ Nível educacional } & $<8$ anos de estudo & 13 & 65,0 & 9 & 45,0 \\
\hline & $\geq 8$ anos de estudo & 7 & 35,0 & 11 & 55,0 \\
\hline \multirow{2}{*}{ Estado civil } & Com companheiro & 19 & 95,0 & 13 & 65,0 \\
\hline & Sem companheiro & 1 & 5,0 & 7 & 35,0 \\
\hline \multirow{3}{*}{ Parentesco com a criança } & Mãe & 16 & 80,0 & 18 & 90,0 \\
\hline & Avó & 3 & 15,0 & 2 & 10,0 \\
\hline & Madrasta & 1 & 5,0 & 0 & - \\
\hline \multirow{4}{*}{ Religião } & Católica & 9 & 45,0 & 7 & 35,0 \\
\hline & Evangélica & 10 & 50,0 & 9 & 45,0 \\
\hline & Outras & 1 & 5,0 & 1 & 5,0 \\
\hline & Não tem & 0 & - & 3 & 15,0 \\
\hline \multirow{3}{*}{ Praticante } & Sim & 11 & 55,0 & 12 & 60,0 \\
\hline & Não & 9 & 45,0 & 5 & 25,0 \\
\hline & Não tem religião & 0 & - & 3 & 15,0 \\
\hline \multirow{4}{*}{ Número de moradores da casa } & 3 moradores & 4 & 20,0 & 4 & 20,0 \\
\hline & 4 moradores & 8 & 40,0 & 6 & 30,0 \\
\hline & 5 moradores & 5 & 25,0 & 5 & 25,0 \\
\hline & 6 ou mais moradores & 3 & 15,0 & 5 & 25,0 \\
\hline \multirow{2}{*}{ Trabalho } & Em casa & 7 & 35,0 & 10 & 50,0 \\
\hline & Fora de casa & 13 & 65,0 & 10 & 50,0 \\
\hline \multirow{4}{*}{ Faixa de renda pessoal } & Sem rendimento & 5 & 25,0 & 5 & 25,0 \\
\hline & Até 1 salário mínimo (SM) & 5 & 25,0 & 6 & 30,0 \\
\hline & Mais de 1 e menos de $2 \mathrm{SM}$ & 9 & 45,0 & 9 & 45,0 \\
\hline & Mais de 2 SM & 1 & 5,0 & 0 & - \\
\hline \multirow{4}{*}{ Faixas de renda familiar em reais } & Mais de 1 e menos de $2 \mathrm{SM}$ & 3 & 15,0 & 10 & 50,0 \\
\hline & Mais de 2 e menos de $3 \mathrm{SM}$ & 9 & 45,0 & 6 & 30,0 \\
\hline & Mais de 3 e menos de $5 \mathrm{SM}$ & 7 & 35,0 & 4 & 20,0 \\
\hline & Mais de $5 \mathrm{SM}$ & 1 & 5,0 & 0 & - \\
\hline
\end{tabular}

diagnosticada com TDAH, como com a criança sem o diagnóstico e sem histórico de doença ou atendimento psicológico, psiquiátrico e/ou neurológico. O questionário contém quatro itens, sendo que o participante deveria responder em uma escala tipo Likert, de 5 pontos, de intensidade variando de "nada estressante" a "muito estressante".

\section{Procedimentos}

O presente estudo foi desenvolvido junto a uma unidade mista de saúde, do serviço público de uma cidade de grande porte. A unidade funciona como: a) Unidade básica de saúde e algumas especialidades para usuários de sua área de abrangência; b) Atenção secundária no atendimento de algumas especialidades para toda a cidade e alguns pequenos municípios próximos.
A coleta do Grupo 1 (clínico) ocorreu no próprio ambulatório, nas condições reais de atendimento. Os participantes do Grupo 2 (comparação), formado a partir do pareamento das crianças do Grupo 1, foram procurados em quatro Unidades Básicas de Saúde.

Inicialmente foi aplicado o Questionário de Capacidades e Dificuldades $(S D Q)$. A partir da avaliação do $S D Q$ e atendimento aos critérios de inclusão, foram aplicados o Inventário de Sintomas de Stress de Lipp (ISSL) e o Questionário de Autopercepção do Estresse.

Os instrumentos foram aplicados individualmente, em entrevista face a face, e tiveram duração de, aproximadamente, uma hora. Procedeu-se ao tratamento estatístico não paramétrico dos dados. Para a comparação entre os grupos foram utilizados o Teste de Mann-Whitney e o Teste Exato de Fisher, 
e adotaram-se valores de $\mathrm{p} \leq 0,05$. O trabalho foi desenvolvido após a aprovação no Comitê de Ética, processo HCRP no 11196/2008, e todos os participantes do estudo concordaram com sua participação através da assinatura do Termo de Consentimento Livre e Esclarecido.

\section{Resultados}

Os resultados obtidos pelo Inventário de Sintomas de Stress para Adultos de Lipp (Lipp, 2000) apontam que 19 dos 20 participantes do G1 (95,0\%) apresentaram indicadores de presença de estresse. Em relação às fases do estresse, $40,0 \%$ se encontraram na fase de resistência, $50,0 \%$ na fase de quase exaustão e 5,0\% na fase de exaustão. Cabe destacar que a sintomatologia predominantemente apresentada pelos participantes do G1 foi de ordem psicológica. No G2, 14 cuidadores $(70,0 \%)$ não apresentaram indicadores de presença de estresse. Dos 6 participantes $(30,0 \%)$ que apresentaram manifestações de estresse, 20,0\% encontraram-se na fase de resistência, $10,0 \%$ na fase de quase exaustão e sintomatologia predominantemente de ordem psicológica.

Os resultados da análise estatística obtidos na comparação entre os grupos, em relação à presença de indicadores de estresse, apontaram que o percentual de cuidadores com indicadores de manifestação de estresse no grupo de crianças com TDAH (G1) foi significativamente maior do que G2 $(\mathrm{p}<0,001)$. Cabe destacar que os cuidadores do G1 apresentaram indicadores de estresse superiores aos obtidos pelos dados normativos do instrumento.

Os resultados, em relação à percepção pessoal dos participantes quanto ao próprio estresse e fatores estressantes, obtidos através do Questionário de Autopercepção em relação ao estresse, apontaram que, entre os cuidadores do G1, $80,0 \%$ se perceberam como estressados ou muito estressados, $45,0 \%$ avaliaram o ambiente da casa como muito estressante e $65,0 \%$ avaliaram o convívio com a criança e os cuidados com a criança como muito estressante. Em relação ao G2, ao contrário, os fatores: ambiente da casa $(50,0 \%)$, convívio com a criança $(75,0 \%)$ e cuidados com a criança $(85,0 \%)$ foram considerados, pela maioria, como nada ou pouco estressante. Para os participantes do grupo 2 predominou a percepção de si como pouco ou nada estressados (75,0\%).

A análise estatística dos dados referentes à percepção pessoal quanto ao estresse e de fatores estressantes apontaram que os fatores convívio com a criança $(p<0,001)$, cuidados com a criança $(p<0,001)$ e percepção do próprio estresse $(\mathrm{p}=0,003)$ apresentaram médias significativamente maiores no Grupo 1, quando comparados com o Grupo 2. Tal dado indica que no grupo de cuidadores de crianças diagnosticadas com TDAH houve maior percepção de estresse no convívio com a criança, no cuidado com a criança e, ainda, que os cuidadores de tal grupo se percebem mais estressados que os do grupo de comparação. Não foram encontradas diferenças estatisticamente significativas em relação ao estresse no ambiente da casa.

Os dados relativos às respostas do $\mathrm{G} 1$ a respeito da identificação de dificuldades da criança (Parte II $S D Q$ ) podem ser visualizados na Tabela 2.

TABELA 2

Frequência (f) e porcentagem (\%) de participantes do Grupo 1 referentes à identificação de dificuldade na criança, obtidos pelo SDQ

\begin{tabular}{|c|c|c|c|}
\hline \multirow{2}{*}{$\begin{array}{l}\text { Dimensões da } \\
\text { dificuldade }\end{array}$} & \multirow{2}{*}{ Especificação } & \multicolumn{2}{|c|}{ Grupo $1(n=20)$} \\
\hline & & $F$ & $\%$ \\
\hline \multirow{4}{*}{$\begin{array}{l}\text { Identificação de } \\
\text { dificuldade }\end{array}$} & Nada & 0 & - \\
\hline & Pequena & 3 & 15,0 \\
\hline & Bem definida & 5 & 25,0 \\
\hline & Grave & 12 & 60,0 \\
\hline \multirow{4}{*}{ Tempo da dificuldade } & Menos de um mês & 0 & - \\
\hline & 1 a 5 meses & 0 & - \\
\hline & 6 a 12 meses & 1 & 5,0 \\
\hline & Mais de um ano & 19 & 95,0 \\
\hline \multirow{4}{*}{ Incomoda a criança } & Nada & 3 & 15,0 \\
\hline & Um pouco & 7 & 35,0 \\
\hline & Muito & 10 & 50,0 \\
\hline & Mais que muito & 0 & - \\
\hline \multirow{4}{*}{$\begin{array}{l}\text { Atrapalha o dia a dia } \\
\text { em casa }\end{array}$} & Nada & 1 & 5,0 \\
\hline & Um pouco & 7 & 35,0 \\
\hline & Muito & 8 & 40,0 \\
\hline & Mais que muito & 4 & 20,0 \\
\hline \multirow{4}{*}{$\begin{array}{l}\text { Atrapalha amizades } \\
\text { da criança }\end{array}$} & Nada & 2 & 10,0 \\
\hline & Um pouco & 6 & 30,0 \\
\hline & Muito & 10 & 50,0 \\
\hline & Mais que muito & 2 & 10,0 \\
\hline \multirow{4}{*}{$\begin{array}{l}\text { Atrapalha aprendizado } \\
\text { escolar }\end{array}$} & Nada & 0 & - \\
\hline & Um pouco & 3 & 15,0 \\
\hline & Muito & 13 & 65,0 \\
\hline & Mais que muito & 4 & 20,0 \\
\hline \multirow{4}{*}{$\begin{array}{l}\text { Atrapalha atividades } \\
\text { de lazer }\end{array}$} & Nada & 7 & 35,0 \\
\hline & Um pouco & 4 & 20,0 \\
\hline & Muito & 8 & 40,0 \\
\hline & Mais que muito & 1 & 5,0 \\
\hline \multirow{4}{*}{$\begin{array}{l}\text { Peso para você ou } \\
\text { família }\end{array}$} & Nada & 4 & 20,0 \\
\hline & Um pouco & 4 & 20,0 \\
\hline & Muito & 6 & 30,0 \\
\hline & Mais que muito & 6 & 30,0 \\
\hline
\end{tabular}




\section{Discussão}

Das crianças em tratamento e que contemplaram os critérios de inclusão para a participação no estudo, $80,0 \%$ foram do sexo masculino. Esse dado encontra-se em consonância com o estudo de prevalência realizado por Biscegli et al. (2013) e corrobora a maior prevalência de diagnóstico do TDAH no sexo masculino, em comparação com o feminino. É possível hipotetizar que desde o início da identificação do problema, pelos pais ou pela escola, os sintomas de agitação e impulsividade, por causarem maior perturbação no ambiente, estejam sendo prioritariamente identificados, principalmente nos meninos.

No presente trabalho, os cuidadores do grupo clínico, em 95,0\% dos casos, apresentaram indicadores de presença de estresse, sendo tal porcentagem significativamente maior quando comparada ao da população em geral, segundo os critérios propostos pelos dados normativos do ISSL, e também quando comparada ao grupo de comparação $(30,0 \%)$. Este dado mostra-se em concordância com estudos que apontam a associação entre estresse e a atividade de ser cuidador de um indivíduo em sofrimento (Del Bianco Faria \& Cardoso, 2010; Santos \& Cardoso, 2015) inclusive o cuidado a uma criança com TDAH (Bellé et al., 2009; Yousefia, Far, \& Abdolahian, 2011).

O convívio com uma criança inclui relacionamento próximo e prestação de cuidados, podendo-se pensar que no contexto de uma criança com TDAH tais atividades tenham caráter mais estressante, devido ao comportamento da criança e do efeito cumulativo da situação, o que acaba por intensificar as dificuldades e estresse vivenciados no dia a dia. As dificuldades dos cuidadores de crianças com TDAH também foram relatadas e discutidas em alguns estudos. Em pesquisa realizada por Bellé et al. (2009), a dimensão do estresse que sobressaiu entre as mães foi a sobrecarga emocional, relativa ao cansaço e à tensão em função das dificuldades para cuidar da criança, preocupação com seu futuro e ressentimento na família.

Ainda em relação ao estresse verificou-se que $50,0 \%$ do grupo clínico encontrou-se na fase de quase exaustão, $40,0 \%$ na fase de resistência e $5,0 \%$ na de exaustão. Esses dados apontaram para a presença de um estresse já instalado, que exigia esforços na tentativa de restabelecer o equilíbrio interno. O predomínio de estresse na fase de quase exaustão aponta para a presença de um esgotamento, na qual os cuidadores estariam apresentando poucos recursos para lidar com as crianças, e se encontrariam vulneráveis ao aparecimento de doenças geneticamente inscritas (Lipp \& Malagris, 1998; Tabaquim \& Marquesini, 2013).
Somado a isso, ressalta-se que a sintomatologia predominante no grupo clínico foi de ordem psicológica. Os cuidadores demonstraram experimentar sentimentos como irritação, depressão, desesperança, hipersensibilidade emotiva, entre outros, indicando a insuficiência de recursos para lidarem com as exigências da situação. Uma vez que a energia para adaptação é finita (Selye, 1983), há a necessidade de que esses cuidadores desenvolvam estratégias para melhor manejar os estressores, pois o gasto de energia enfraquece o organismo, colocando-o vulnerável e aumenta a suscetibilidade do indivíduo às doenças. Cabe enfatizar, assim, que essa sintomatologia influencia negativamente na saúde do cuidador, na capacidade desses em oferecer acolhimento e responder às demandas da criança e em sua qualidade de vida (Lipp \& Malagris, 1998).

Destaca-se que a totalidade dos cuidadores primários das crianças participantes do estudo foi do sexo feminino o que está em concordância com outros estudos que indicam a mulher como a principal responsável pelo cuidado (Romagnoli, 2006; Santos \& Cardoso, 2015;). Dentre as cuidadoras, a maioria, tanto no grupo de comparação ( $\mathrm{G} 2=90,0 \%)$, quanto no grupo clínico $(\mathrm{G} 1=80,0 \%)$ foram as mães das crianças, corroborando outros trabalhos de investigação (Estevam, Marcon, Antonio, Munari \& Waidman, 2011; Narkunam, Hashim, Sachdev, Pillai \& Guan Ng, 2014; Santos \& Cardoso, 2015) Somado a esses dados observou-se que as cuidadoras das crianças do G1 possuiam até 40 anos de idade $(55,0 \%)$, pouca escolaridade $(65,0 \%$ com menos de 8 anos de estudo), a maioria trabalhava fora de casa $(65,0 \%)$ e $95,0 \%$ delas recebiam menos de dois salários mínimos. Pode-se refletir sobre o papel que é depositado e assumido dentro da família pela mulher no cuidado com os filhos e a sobrecarga advinda por ter responder, além das demandas domésticas de cuidado à família, as demandas do trabalho, caracterizando desse modo uma dupla jornada. Aponta-se para a vulnerabilidade dessas cuidadoras, o que potencializa o estresse e dificulta seu manejo, tendo em vista as precárias condições econômicas e sociais e o acúmulo de funções que executam.

Em relação à autopercepção de estresse e de fatores estressantes observou-se que no Grupo 1 houve maior percepção de estresse no convívio e cuidado com a criança e, ainda, que os cuidadores desse grupo se perceberam mais estressados que os do Grupo 2, indicando que a avaliação em relação ao próprio estresse do grupo de cuidadores de crianças diagnosticadas com TDAH foi significativamente maior e que o mesmo estava vinculado predominantemente ao convívio e cuidado com a criança. 
Os resultados relativos à segunda parte do SDQ, respondidos pelos cuidadores do grupo clínico, complementaram e ajudaram a compreender essas respostas. Observou-se que, para a maioria dos cuidadores, a dificuldade foi identificada como grave, existindo há mais de um ano e incomodando a eles e à criança. Cabe ressaltar que a avaliação subjetiva que o indivíduo faz de determinada situação, sua percepção, interpretação e sentido, influenciarão na forma de lidar com tal situação e, ainda, na avaliação acerca dos recursos pessoais para enfrentá-la (Regalla, Guilherme, $\&$ Serra-Pinheiro, 2007). Nesse sentido, pode-se pensar na sensação de desesperança e possivelmente de impotência vivida pelos cuidadores, uma vez que são cuidadores com altos níveis de estresse, percebendose estressados, identificando a situação como grave e persistente.

Os cuidadores relataram também que a dificuldade da criança atrapalhava muito o dia a dia em casa, atrapalhava as amizades da criança, o aprendizado escolar e a realização de atividades de lazer, o que demonstrou que diversos aspectos de suas vidas estavam sendo impactados pelo transtorno. Ainda na segunda parte do SDQ, 30\% dos cuidadores consideraram que a criança é muito peso e $30,0 \%$ mais que muito peso para eles ou para a família como um todo. "Muito peso" sugere a existência de demandas excessivas que não podem ser cumpridas pelos cuidadores excedendo as suas capacidades para lidar com a situação. Nesse sentido, pode-se supor a existência de dificuldades nos vínculos entre crianças e cuidadores, mantidas através de processos de retroalimentação de forma que o comportamento difícil da criança afeta emocionalmente os cuidadores, e vice-versa, formando uma relação circular.

Diante do exposto, verificou-se que o cuidador primário estava vivenciando o contato e convívio com a criança com TDAH com muitas dificuldades, com altos níveis de estresse e esgotamento emocional, percebendo-se estressado, atribuindo isto, principalmente, ao convívio e cuidado à criança. No entanto, cabe ressaltar que não foi efetuado um estudo longitudinal e assim, não se sabe como se estabeleceram as relações entre cuidador e criança, e nem se os altos níveis de estresse desses cuidadores já estavam presentes no início do contato com as crianças. Neste sentido, estudos apontam a dificuldade de afirmar se a disfunção familiar é a causa ou o efeito do TDAH, pois existem dificuldades nos vínculos entre as crianças e os cuidadores primários, mantidas através de processos de retroalimentação (D'avila-Bacarji et al., 2005). Assim, o contexto social em que a criança vive pode funcionar tanto como desencadeador ou potencializador de diversas perturbações, quanto pode conter, ou mesmo minimizar dificuldades enfrentadas pelas crianças. Nunes e Werlang (2008) apontam a necessidade de se entender o indivíduo como parte de um sistema, com elementos que interagem, influenciando cada parte e sendo por ela influenciados.

No entanto, apesar dessas dificuldades poderem estar presentes na vida desta dupla há mais tempo, há a necessidade de ações considerarem o momento atual. Através da presente pesquisa, pôde-se verificar que as crianças apresentaram dificuldades emocionais e comportamentais e os cuidadores podiam estar vivenciando dificuldades em tolerar, em saber como lidar, cansaço e esgotamento diante de situações que não conseguiam administrar. Pôde-se supor, ainda, que as dificuldades vivenciadas pelos cuidadores tinham impacto também na sua vida social, limitando as oportunidades de encontros, trocas e apoio social. Vale ressaltar que os locais que poderiam compartilhar o cuidado, como a escola e a rede de amizades da criança, normalmente queixam-se da mesma e "pressionam" os familiares em busca de solução para o comportamento disruptivo da criança. Supõe-se, assim, um certo isolamento do cuidador e da criança, contribuindo para as manifestações de estresse.

Nesse contexto, aponta-se a necessidade de auxiliar essas famílias no desenvolvimento de recursos para lidar com essas situações, tais como: orientações, informações, apoio social e emocional, espaços de convivência e escuta, grupos entre cuidadores e atividades para as crianças. Entretanto, observou-se que o tratamento recebido pelas crianças do grupo clínico no serviço de saúde foi prioritariamente o atendimento neurológico à criança, poucas com atendimento psicológico, psicopedagógico, sendo que a maioria $(70,0 \%)$ foi medicada e, ainda, ressalta-se que as intervenções realizadas pelo serviço não incluíram o cuidador. Aponta-se assim que a terapêutica estava centrada no tratamento individual, focado nos aspectos biológicos. O tratamento do TDAH, baseado principalmente na terapêutica medicamentosa, adotado muitas vezes como forma única de intervenção, foi apontado em alguns trabalhos (Caliman, 2008; Pereira \& Silva, 2011; Bianchi \& Faraone, 2015).

Embora não tenha sido objeto de estudo desta investigação, esses resultados relativos ao tratamento ofertado pelo serviço de saúde, que se encontra no nível secundário do sistema de saúde, possibilitaram apontar a necessidade de ampliar o cuidado, englobando o contexto onde surge determinada queixa, considerando a realidade familiar, social e comunitária, pois é nesses ambientes que se processam as necessidades de saúde do indivíduo e, onde elas podem ser cuidadas e até 
mesmo sanadas (Resta \& Motta, 2005). Como exemplo tem-se as iniciativas de acompanhamento terapêutico, em que o profissional caminha junto com o usuário seguindo-o nas adversidades, e podendo contribuir para que o mesmo continue fazendo trocas sociais mesmo que em sofrimento e construa novas possibilidades de vida (Gonçalves, 2011). E ainda, propostas grupais com os cuidadores, no qual os familiares pudessem relatar suas próprias experiências, medos, fantasias e onde as dúvidas pudessem ser refletidas conjuntamente e possivelmente respondidas, possibilitando trocas e ampliando a rede social e o aprendizado no contato com o outro.

Destaca-se, que tais ações poderiam auxiliar o cuidador a desenvolver maiores recursos internos, e ainda, proporcionar a utilização de formas mais eficientes de estratégias para enfrentar a situação. O presente trabalho, ao estudar estresse e a autopercepção de estresse em cuidadores de crianças com TDAH possibilitou ampliar o conhecimento acerca desses cuidadores, no seu próprio contexto e especificidades e, ainda, identificar possíveis riscos psicossociais.

\section{Considerações Finais}

Ao analisar os dados obtidos através da pesquisa, nota-se que os cuidadores de criança com TDAH eram mulheres, principalmente mães, jovens, de baixa renda e escolaridade e que trabalhavam fora de casa e, portanto, acumulavam diversas funções. Tais cuidadoras apresentaram altos níveis de estresse e esgotamento emocional, o que tem provocado impacto e perturbações na vida familiar. Nesse contexto, avaliar o estresse, a auto-percepção do estresse e de fatores estressantes se mostrou relevante na medida em que ampliou o conhecimento do contexto no qual a criança vive, possibilitando identificar algumas dificuldades e impactos do sofrimento da criança e de seu cuidador na vida dos mesmos.

Encontrou-se no presente estudo, que as crianças que recorreram ao serviço receberam um tratamento baseado, principalmente, numa terapêutica individual, biológica e medicamentosa. Ressalta-se que cuidar dos aspectos físicos e biológicos dos usuários em tratamento é de extrema importância, no entanto, faz-se necessário valorizar o contexto emocional e social. Os dados relativos aos cuidadores, referentes às manifestações de estresse, apontam o quanto tais intervenções são reducionistas frente às demandas da criança e de sua família. Nesse sentido, sugere-se que os serviços incluam a família no projeto terapêutico à criança, estabelecendo uma parceria que favoreceria um cuidado integral à família.

Analisando-se alguns limites do presente estudo pode-se considerar que a faixa etária estudada foi ampla e também não restringiu o gênero das crianças. Por se tratar de um estudo desenvolvido no contexto de um serviço de saúde, sem qualquer interferência na dinâmica dos atendimentos, não foi possível restringir mais a faixa etária, nem o gênero das crianças. Foi priorizada a presença do diagnóstico de TDAH pelo médico responsável sem, no entanto, ter iniciado o uso de medicação e pontuação no SDQ, na escala específica para hiperatividade na faixa anormal. Com estes critérios pode-se, dentro de determinados limites, controlar a presença de comportamentos que poderiam ter relação com outros fatores que não o TDAH.

Diante desse cenário complexo em que se mostrou o do TDAH, aponta-se a necessidade de novos estudos que acompanhem a criança e sua família, de forma longitudinal, buscando compreender a dinâmica envolvida em tal relação, pois o contexto social em que a criança vive pode funcionar como desencadeador ou potencializador de diversas perturbações. Acredita-se também serem necessários novos estudos que sistematizem e avaliem os serviços de saúde. Tais estudos poderão auxiliar numa melhor adequação dos serviços às necessidades das crianças e familiares, apontando indicadores importantes para a reestruturação dos mesmos e instrumentalizando os profissionais de saúde para o cuidado.

Conclui-se que, são familiares cuidadores apresentando poucos recursos emocionais, econômicos, diante de crianças com dificuldades comportamentais e emocionais, que demandam muita atenção e cuidados. E ainda, tais cuidadores possuem outras responsabilidades as quais necessitam corresponder, como por exemplo, a necessidade de conciliar o trabalho fora de casa com os cuidados à família, o que dificulta o enfrentamento da situação. Desse modo, iniciativas de cuidado ao cuidador são necessárias, com investimentos em programas de intervenção voltados ao cuidado integral à família, que poderão instrumentalizá-la quanto ao manejo de situações estressoras, bem como quanto às exigências advindas do papel de cuidador. Tais ações poderão refletir na melhora do tratamento da criança, bem como fortalecer os vínculos de convivência entre todos os integrantes da família. 


\section{Referências}

American Psychiatric Association (2002). Manual Diagnóstico e Estatístico de Transtornos Mentais (4aa ed., rev.) (DSM-IV-TR). Porto Alegre: Artmed.

Bargas, J. A. \& Lipp, M. E. N. (2013). Estresse e estilo parental materno no transtorno de déficit de atenção e hiperatividade. Psicologia Escolar e Educacional, 17(2), 205-213. http://dx.doi.org/10.1590/S1413-85572013000200002

Bellé, A. H., Andreazza, A. C., Ruschel, J., \& Bosa, C. A. (2009). Estresse e adaptação psicossocial em mães de crianças com Transtorno de déficit de atenção/hiperatividade. Psicologia: Reflexão e Crítica, 22 (3), 317-325. http://dx.doi. org/10.1590/s0102-79722009000300001

Bianchi, E. \& Faraone, S. A. (2015). El Trastorno por Déficit de Atención e Hiperactividad (TDA/H). Tecnologías, actores sociales e industria farmacêutica. Physis: Revista de Saúde Coletiva, 25(1), 75-98.

Biscegli, T. S., Galego, A. R, Galdezzani, J. P., Faria, T. S., Galego, D. R. C., Felipe, B. (2013). Prevalência de transtorno de déficit de atenção e hiperatividade (TDAH) em pré-escolares de creches. Pediatria Moderna, 49(8), 330-336.

Caliman, L. V. (2008). O TDAH: entre as funções, disfunções e otimização da atenção. Psicologia em Estudo, 13(3), 559-566. http://dx.doi.org/10.1590/S1413-73722008000300017

D'avila-Bacarji, K. M. G., Marturano, E. M., \& Elias, L. C. S. (2005). Suporte parental: um estudo sobre crianças com queixas escolares. Psicologia em Estudo (Maringá), 10(1), 107-115. http://dx.doi.org/10.1590/S141373722005000100013

Del Bianco Faria, A. M. \& Cardoso, C. L. (2010). Aspectos psicossociais de acompanhantes cuidadores de crianças com câncer: stress e enfrentamento. Estudos de Psicologia (Campinas), 27(1), 13-20. http://dx.doi.org/10.1590/S0103166X2010000100002

Estevam, M. C., Marcon, S. S., Antonio, M. M., Munari, D. B., \& Waidman, M. A. P. (2011). Convivendo com transtorno mental: perspectiva de familiares sobre atenção básica. Revista da Escola de Enfermagem da USP, 45(3), 679-686. http://dx.doi.org/10.1590/S0080-62342011000300019

Fleitlich-Bilyk, B. W. (2002). The prevalence of psychiatric disorders in 7-14-year olds in the southeast of Brazil [Tese de doutorado]. Londres: Department of Child and Adolescent Psychiatry. Institute of Psychiatry. King's College. London University.

Gonçalves, C. A. B. (2011). O acompanhamento terapêutico e a reforma psiquiátrica. In A. E. A. Antunez (Org.), Acompanhamento terapêutico: casos clínicos e teoria (pp. 21-40). São Paulo: Casa do Psicólogo.

Goodman, R. (1997) The Strenghts and Difficulties Questionnaire: a research note. Journal of Child Psychology and Psychiatry, 38(5), 581-586. http://dx.doi.org/10.1111/j.1469-7610.1997.tb01545.x

Hora, A. F., Silva, S., Ramos, M., Pontes, F., \& Nobre, J. P. (2015). The prevalence of ADHD: a literature review. Psicologia, 29 (2), 47-62. http://dx.doi.org/10.17575/rpsicol.v29i2.1031

Lipp, M. E. N. (2000). Manual do Inventário de Sintomas de Stresse para adultos de Lipp (ISSL) - manual. São Paulo: Casa do Psicólogo.

Lipp, M. E. N. \& Malagris, L. N. (1998). Manejo do estresse. In B. Rangé (Org.), Psicoterapia comportamental e cognitiva: pesquisa, prática, aplicações e problemas (pp. 279-292). São Paulo: PSY.

Marini, A. M., Martins, M. R. I., Vigãno, A., Filho, A. B. M., \& Pontes, H. E. R. (2010). Sobrecarga de cuidadores na psiquiatria infantil. Revista Neurociencias, 18, 300-306. Recuperado de: http://www.revistaneurociencias.com.br/ edicoes/2010/RN1803/477\%20original.pdf

Narkunam, N., Hashim, A. H., Sachdev, M. K., Pillai, S. K., \& Guan Ng, C. (2014). Stress among parents of children with attention deficit hyperactivity disorder, a Malaysian experience. Asia-Pacific Psychiatry, 6, 207-216.

Nunes, M. M. S. \& Werlang, B. S. G. (2008). Transtorno de Déficit de Atenção/Hipertividade e Transtorno de Conduta: aspectos familiares e escolares. Conscientiae Saúde, 7, 207-216. http://dx.doi.org/10.5585/conssaude.v7i2.970

Oliveira, C. G. \& Albuquerque, P. B. (2009). Diversidade de Resultados no Estudo do Transtorno de Déficit de Atenção e Hiperatividade. Psicologia: Teoria e Pesquisa, 25(1), 93-102. http://dx.doi.org/10.1590/s0102-37722009000100011

Pereira, I. S. A. \& Silva, J. C. (2011). Transtorno de déficit de atenção/hiperatividade à luz de uma abordagem crítica: um estudo de caso. Psicologia em Revista, 17(1), 117-134.

Petresco S., Anselmi L., Santos I. S., Barros A. J., Fleitlich-Bilyk B., Barros F. C., \& Matijasevich, A. (2014). Prevalence and comorbidity of psychiatric disorders among 6-year-old children: 2004 Pelotas Birth Cohort. Social Psychiatry and Psychiatric Epidemiology, 49, 975-83. http://dx.doi.org/10.1007/s00127-014-0826-Z

Pires, T. de O., Silva, C. M. F. P. da, \& Assis, S. G. de. (2012). Ambiente familiar e transtorno de déficit de atenção e hiperatividade. Revista de Saúde Pública, 46(4), 624-633. http://dx.doi.org/10.1590/S0034-89102012005000043

Regalla, M. A., Guilherme, P. R., \& Serra-Pinheiro, M. A. (2007). Resiliência e transtorno do déficit de atenção/ hiperatividade. Jornal Brasileiro de Psiquiatria, 56, 45-49. http://dx.doi.org/10.1590/S0047-20852007000500010

Resta, D. G. \& Motta, M. G. C. (2005). Família em situação de risco e sua inserção no programa de saúde da família: uma reflexão necessária à prática profissional. Texto \& Contexto Enfermagem, 14, 109-115. http://dx.doi.org/10.1590/ S0104-07072005000500014

Rodhe, L. A., Busnello, E. A., Chachamovitch, E., Vieira, G. M., Pinzon, V., \& Kelzer, C. R. (1998). Transtorno de déficit de atenção/hiperatividade: revisando conhecimentos. Revista Brasileira de Psiquiatria, 20, 166-178. 
Romagnoli, C. (2006). Famílias na rede de saúde mental: um breve estudo esquizoanalítico. Psicologia em Estudo, 11(2), 305-314. http://dx.doi.org/10.1590/S1413-73722006000200009

Santos, A. F. O. \& Cardoso, C. L. (2015). Family members of individuals suffering from mental disorders: stress and care stressors. Estudos de Psicologia (Campinas), 32(1), 87-95. http://dx.doi.org/10.1590/0103-166X2015000100008

Santos, L. F. \& Vasconcelos, L. A. (2010). Transtorno do déficit de atenção e hiperatividade em crianças: uma revisão interdisciplinar. Psicologia: Teoria e Pesquisa (Brasília), 26(4), 717-724. http://dx.doi.org/10.1590/s010237722010000400015

Schroeder, V. M. \& Kelley, M. L. (2009) Associations between family environment, parenting practices, and executive functioning of children with and without ADHD. Journal of Child and Family Studies, 18(2), 227-35. http://dx.doi. org/10.1007/s10826-008-9223-0

Selye, H. (1956). The stress of life. New York: Longmans.

Selye, H. (1983). The Stress Concept: past, present and future. In C. L. Cooper (Ed.), Stress research: issues for the eighties (pp. 1-20). USA: John Wiley \& Sons.

Silva, F. M. \& Correa, I. (2006). Doença crônica na infância: vivência do familiar na hospitalização da criança. REME Revista Mineira de Enfermagem, 10(1), 18-23.

Stone, R., Cafferata, G. L., \& Sangl, J. (1987). Caregivers of the frail elderly: a national profile. The Gerontologist (Washington), 27(5), 616-626. http://dx.doi.org/10.1093/geront/27.5.616

Tabaquim, M. L. M. \& Marquesini, M. A. M. (2013). Study of parentes of patients with cleft lip and palate in a surgical process. Estudos de Psicologia (Campinas), 30(4), 517-524. http://dx.doi.org/10.1590/S0103-166X2013000400005

Yousefia, S., Far, A. S., \& Abdolahian, E. (2011). Parenting estresse and parenting styles in mothers of ADHD with mothers of normal children. Procedia - Social and Behavioral Sciences, 30, 1666-1671. http://dx.doi.org/10.1016/j. sbspro.2011.10.323

\footnotetext{
Autores:

Ana Maria Del Bianco Faria - Mestre, Centro Universitário UNIFAFIBE.

Carmen Lucia Cardoso - Doutora, Professora do Departamento de Psicologia, Faculdade de Filosofia, Ciências e Letras de Ribeirão Preto, Universidade de São Paulo.
}

Endereço para correspondência:

Carmen Lúcia Cardoso

Av. Bandeirantes, 3900

14040-901 Ribeirão Preto, SP, Brasil

<carmen@ffclrp.usp.br>

Recebido em: 04.08.2015

Aceito em: 13.05.2016 